\title{
Changes in Muscle Activity of the Serratus Anterior According to Surface Tilt Angle During Push-up Plus Exercise in Subjects With Winged Scapula
}

\author{
Qian Gu ${ }^{1}, \mathrm{MSc}, \mathrm{PT}, \mathrm{Tae}-\mathrm{ho} \mathrm{Kim}^{2}, \mathrm{PhD}, \mathrm{PT}, \mathrm{Jung}-\mathrm{genn} \mathrm{Chun}^{3}$, MSc, PT \\ ${ }^{1}$ Dept. of Rehabilitation Medicine, Affiliated Hospital of Nantong University, China \\ ${ }^{2,3}$ Dept. of Physical Therapy, Daegu University, Republic of Korea
}

\begin{abstract}
Background: The serratus anterior is one of the most important muscle for maintaining good scapular alignment in the shoulder joint. The pectoralis major and upper trapezius may also compensate for weak serratus anterior muscles. The push-up plus exercise has been identified as the optimal exercise for maximum activation of the serratus anterior.

Objects: The purpose of this study was to examine differences in surface electromyography (EMG) activity of upper trapezius, pectoralis major, and serratus anterior muscles during push-up plus exercises on variously angled surfaces in subjects with winged scapula.

Methods: Sixteen subjects with winged scapula (male $=5$, female $=11$ ) volunteered for this study. The subjects performed push-up plus exercise on four different tilt angles, namely $0^{\circ}, 30^{\circ}, 60^{\circ}$, and $90^{\circ}$. EMG activities in the serratus anterior, upper trapezius, and pectoralis major muscles during performance of push-up plus exercise were measured in all subjects. Data were processed from repeated measures one-way analysis of variance.

Results: There was significant difference in the muscle activity of the serratus anterior on the different surface angles $(\mathrm{p}<.05)$. The results of the post-hoc analysis showed significantly greater serratus anterior muscle activity on a surface at a $0^{\circ}$ angle than at others tilt angles $(\mathrm{p}<.05)$. There was also significant difference in the ratio of serratus anterior to upper trapezius and serratus anterior to pectoralis major across the four surfaces $(\mathrm{p}<.05)$, and post-hoc analysis showed significantly greater values on the $0^{\circ}$ surface than on other tilts $(\mathrm{p}<.05)$.

Conclusion: This study found that performing push-up plus exercises on a flat surface with $0^{\circ}$ and $30^{\circ}$ tilt angle achieves high activation of the serratus anterior muscle for selective strengthening. It can also take into account the sequential application, which is first performed at a $30^{\circ}$ and at a $0^{\circ}$ tilt angle for and effective but not excessive muscle activation.
\end{abstract}

Key Words: Push-up plus; Serratus anterior; Surface tilt angle; Winged scapula.

\section{Introduction}

Normal scapula movement in shoulder motion consists of six types: elevation, depression, retraction, protraction, and downward and upward rotation (Lawrence et al, 2014; Ludewig et al, 2009; McClure et al, 2001). Both the serratus anterior and trapezius muscles act to stabilize the scapula to the chest wall and are considered the major upward rotators of the scapula (Ebaugh et al, 2005). The pectoralis major and serratus anterior muscles can be activated simultaneously during active scapular exercises (Kim et al, 2010). The high pectoralis major activity during push-up plus exercises can be explained by characteristics of pectoralis major as a synergist and the reduced activation of the serratus anterior (Park

Corresponding author: Tae-ho Kim, hohoho90@naver.com 


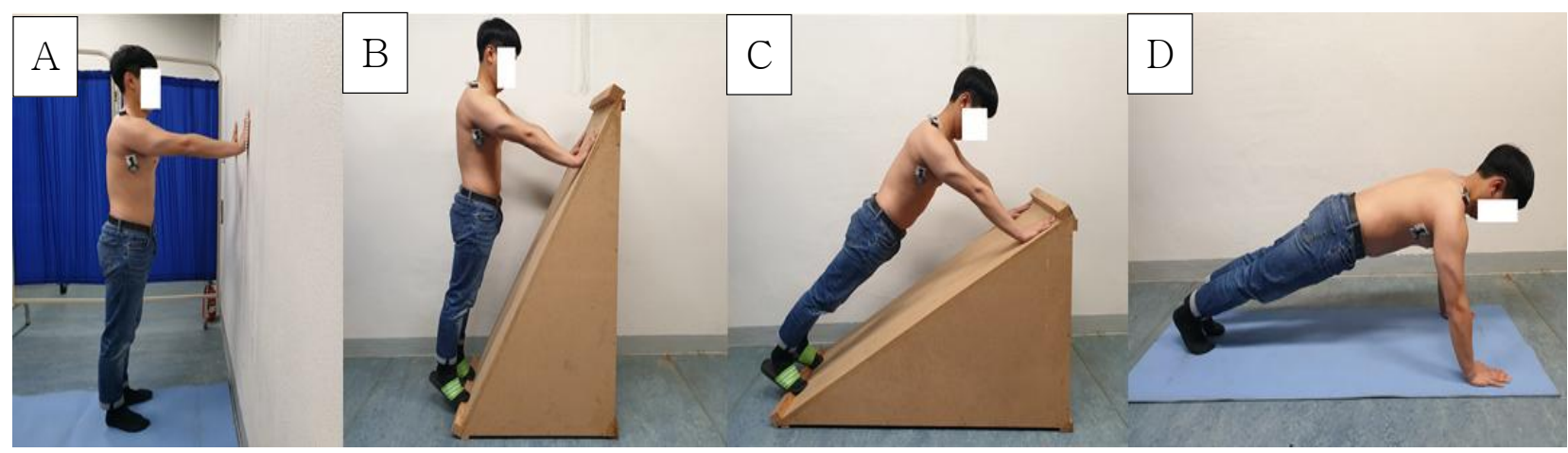

Figure 1. Push-up plus exercise on four surface tilt angles $\left(A: 90^{\circ}, B: 60^{\circ}, \mathrm{C}: 30^{\circ}, \mathrm{D}: 0^{\circ}\right.$ ).

et al, 2014). In some cases, excessive activation of the pectoralis major can lead to glenohumeral and scapulothoracic pathologies such as anterior translation of shoulder joint or decreased compression forces on the glenoid joint (Labriola et al, 2005).

The serratus anterior is one of the most important muscle for maintaining good scapular alignment in the shoulder joint (Kim and Park, 2018), and it is a key component of therapeutic exercise protocols for the prevention and rehabilitation of scapular winging (Park et al, 2013). Therefore, weakness, fatigue, or abnormal muscle firing patterns of the serratus anterior can cause the shoulder to deviate from normal scapulohumeral rhythms and lead to shoulder dysfunction (Madeleine et al, 2008).

Many studies have investigated serratus anterior activation during push-up exercises (Decker et al, 1999; Kim and Park, 2018; Ludewig et al, 2004). The standard push-up plus has been identified as the optimal exercise for maximum activation of the serratus anterior with minimal activation of the upper trapezius when compared to other exercises (Ludewig et al, 2004). Despite the advantages of push-up plus exercise, some patients with shoulder joint dysfunction may not be able to perform or maintain this exercise position due to the gravity and excessive weight load (Kim and Park, 2018). If the surface tilt angles is different during push-up plus exercises, the degree of gravity and weight load will change accordingly. In addition, the muscle activation of shoulder joint will be affected by surface tilt angles. However, changes to serratus anteior, upper trapezius, and pectoralis major activity during push-up plus exercise because of different surface tilt angles are unclear. The purpose of this study was, therefore, to examine differences in activity of these three muscles as well as the ratio of serratus anterior to upper trapezius and serratus anterior to pectoralis major during push-up plus exercises on variously angled surfaces. This study aims to suggest an appropriate surface tilt angle that does not overload patients with shoulder joint dysfunction that require selective activation of serratus anterior.

\section{Methods}

\section{Subjects}

This study was conducted with 16 subjects who have winged scapula attending university students in Gyeongsan, Korea. The mean characteristics of the participants were age of $23.7 \pm 1.7$ years, height of $166.0 \pm 7.8 \mathrm{~cm}$, weight of $56.1 \pm 8.6 \mathrm{~kg}$, and winging distance of $23.31 \pm 1.78 \mathrm{~mm}$. A distance equal to or greater than $20 \mathrm{~mm}$ was defined as winged (Weon et al, 2011). The exclusion criteria were past or present musculoskeletal conditions affecting the shoulder joint or rotator cuff muscles and/or neurological disorders. Prior to participation, all subjects read and signed the university-approved human subjects consent form, and the study was approved by the Daegu University Institutional Review Board (approval 
Table 1. Muscle activities according to surface tilt angles

\begin{tabular}{ccccccc}
\hline \hline Muscles & $90^{\circ}$ & $60^{\circ}$ & $30^{\circ}$ & $0^{\circ}$ & $\mathrm{F}$ & $\mathrm{p}$ \\
\hline $\mathrm{UT}^{\mathrm{a}}$ (\%MVIC) & $9.73 \pm 4.27^{\mathrm{b}}$ & $9.11 \pm 7.28$ & $10.67 \pm 7.20$ & $8.42 \pm 3.53$ & .43 & .732 \\
$\mathrm{PM}^{\mathrm{c}}$ (\%MVIC) & $16.19 \pm 9.08$ & $15.54 \pm 8.01$ & $20.20 \pm 9.09$ & $21.47 \pm 9.78$ & 3.45 & .070 \\
$\mathrm{SA}^{\mathrm{d}}$ (\%MVIC) & $31.33 \pm 11.88$ & $31.32 \pm 13.28$ & $49.38 \pm 17.30$ & $65.17 \pm 13.52$ & 21.28 & $.000^{*}$ \\
$\mathrm{SA} / \mathrm{UT}^{\mathrm{e}}$ & $3.76 \pm 1.99$ & $4.32 \pm 2.33$ & $6.24 \pm 4.08$ & $9.16 \pm 4.41$ & 8.30 & $.000^{*}$ \\
$\mathrm{SA} / \mathrm{PM}^{\mathrm{f}}$ & $2.50 \pm 1.50$ & $2.54 \pm 1.63$ & $3.05 \pm 1.71$ & $3.82 \pm 1.93$ & 10.43 & $.005^{*}$ \\
\hline
\end{tabular}

apper trapezius, ${ }^{\mathrm{b}}$ mean \pm standard deviation, ${ }^{\mathrm{c}}$ pectoralis major, ${ }^{\mathrm{d}}$ serratus anterior, ${ }^{\mathrm{e}}$ ratio of serratus anterior to upper trapezius, ${ }^{\mathrm{f}}$ ratio of serratus anterior to pectoralis major, ${ }^{*} \mathrm{p}<.05$

number: 1040621-201411-HR-013-02).

\section{Instruments}

Winged scapula was confirmed by measuring the distance between the spinous process of the thoracic spine, at the subscapular level, and the inferior angle of the scapula using a digital caliper. Muscle activity during the push-up plus exercises was collected using a wireless surface electromyography (EMG) system (TeleMyo DTS, Noraxon Inc., Scottsdale, AZ, USA). The inclined surface was made from wood constructed as a right-angled triangle with $30^{\circ}, 60^{\circ}$, and $90^{\circ}$ tilt angles (length $\times$ width $\times$ height $=138 \times$ $60 \times 80 \mathrm{~cm}$ ) (Figure 1).

\section{Outcome Measures}

Muscle activity was measured using wireless EMG, and electrodes were attached to the muscle belly of the serratus anterior (on the midaxillary line of the right fifth rib) and upper trapezius (in the region of trapezius insertion at the midpoint between the $\mathrm{C} 7$ spinous process and the right acromioclavicular joint) and approximately $2 \mathrm{~cm}$ out from the axillary fold of the pectoralis major (Cram et al, 1998). The average of a root-mean-square (moving window $250 \mathrm{~ms}$ ) values in the EMG data were calculated to quantify the amplitude of the signals using middle three of the five seconds recorded for each of the three muscles, excluding the initial and final seconds during push-up plus exercises. MyoResearch XP Master (version 1.08) software was used to process the data. To normalize the EMG results, maximal voluntary isometric contraction (MVIC) activity was measured for the tested muscles. Each subject was asked to voluntarily and maximally contract each of the muscles for 5 seconds against the manual resistance of the examiner. The MVIC value was derived from the average RMS across three trials, and average EMG activity during push-up plus exercise was expressed as a percentage of the MVIC value (\%MVIC).

\section{Procedures}

The subjects performed push-up plus exercises on four different tilt angles, namely $0^{\circ}, 30^{\circ}, 60^{\circ}$, and $90^{\circ}$. As a starting position, the subjects placed their hands and feet shoulder width apart, placed their head, trunk, and hips in a straight line, pushed their hands toward the surface, and maintained this position for five seconds (Figure 1). The examiner instructed with verbal command that "push your hands in push-up posture as for as possible from the ground, hold for 5 seconds." Each subject repeated the exercise three times. If the subject did not maintain the protraction of starting position in push-up plus, the subject was performed again after sufficient rest.

\section{Statistical Analyses}

Repeated measures one-way analysis of variance was used to compare differences in the muscle activity of the upper trapezius, pectoralis major, and serratus anterior muscles during the push-up plus exercises on the four different tilt angles. Post-hoc analysis was performed by Bonferroni test, and all statistical analyses were conducted using SPSS ver. 


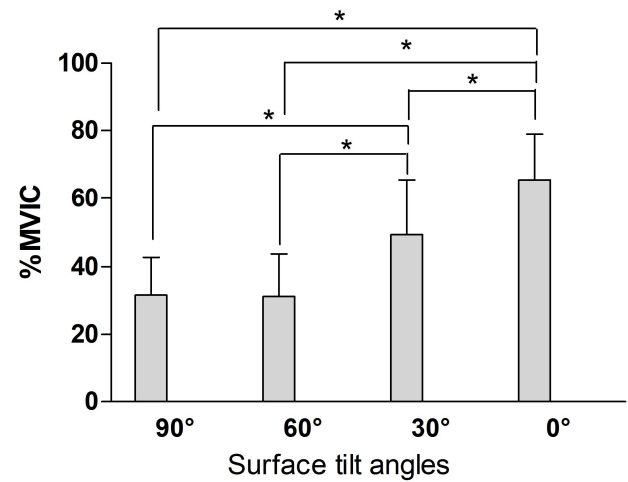

Figure 2. Comparison of serratus anterior activity on surface tilt angles $\left({ }^{*} \mathrm{p}<.05\right)$.

22.0 for Windows (IBM corp., Armonk, NY, USA). Statistical significance was set at $\mathrm{p}<.05$.

\section{Results}

There was significant difference in the muscle activity of the serratus anterior on the different surface angles $(\mathrm{p}<.05) \quad$ (Table 1$)$. The results of the post-hoc analysis showed significantly greater serratus anterior muscle activity on a surface at $0^{\circ}$ and $30^{\circ}$ angle than at others tilt angles $(\mathrm{p}<.05)$ (Figure 2 ). There was also significant difference in the ratio of serratus anterior to upper trapezius across the four surfaces $(\mathrm{p}<.05)$ (Table 1$)$, and post-hoc analysis showed significantly greater values on the $0^{\circ}$ and $30^{\circ}$ surface than on other tilts $(\mathrm{p}<.05)$ (Figure 3 ). There was significant difference in the ratio of serratus anterior to pectoralis major across the four surfaces $(\mathrm{p}<.05) \quad($ Table 1$)$, and post-hoc analysis showed significantly greater values on the $0^{\circ}$ surface than on other tilts $(\mathrm{p}<.05)$ (Figure 3$)$.

\section{Discussion}

The purpose of this study was to examine differences in activity of the upper trapezius, pectoralis major, and serratus anterior muscles during push-up plus exercises with changes to the surface angle.

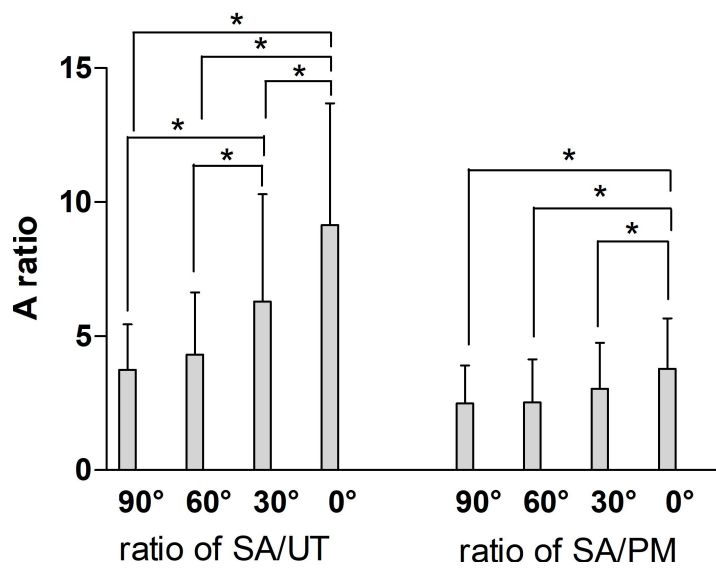

Figure 3. A ratio of serratus anterior to trapezius (left) and a ratio of serratus anterior to pectoralis major (right) $\left({ }^{*} \mathrm{p}<.05\right)$.

Piraua and colleagues (2014) recommended that if the goal of an exercise program is to strengthen the serratus anterior, push-up exercise have been suggested on a flat surface. Furthermore, push-up plus exercises can emphasize scapular protraction and elicit the greatest serratus anterior muscle activity (Ludewig et al, 2004).

The muscle activity of the serratus anterior in this study was significantly increased according to a decline of the surface tilt; at $0^{\circ}$ and $30^{\circ}$, muscle activity was higher than at $60^{\circ}$ and $90^{\circ}$ tilt angles. Ludewig and colleagues (2004) explain that serratus anterior activity increases by more than 20\%MVIC because upward rotation of the scapula should be maintained during exercise against gravity (Ludewig et al, 2004). Therefore, the results of the present study could be explained by weight bearing and gravity. This study demonstrated high activation of the serratus anterior muscle at a $0^{\circ}$ surface tilt angle during push-up plus exercises, and this result agrees with those of previous studies recommending this exercise for winged scapula (Lear and Gross, 1998).

On the other hand, there was no significant difference in activity of the upper trapezius or pectoralis major muscles with the decline in surface tilt. The activity of pectoralis major increased as the surface angle decreased but was not significant. This is thought to increase due to the angle of gravity and 
weight loading, but due to the activity of serratus anterior, it is thought the role of pectoralis major is to be responsible for maintaining the horizontal adduction during push-up plus exercise. Park and colleagues (2014) found that there was no difference in the activity of pectoralis major at $0^{\circ}$ and $90^{\circ}$ surface tilt during push-up plus exercises. The upper trapezius is a synergist for protraction. It seems that the agonist, serratus anterior, is greatly activated, the upper trapezius do not contribute much to protraction during push-up plus exercise.

High ratios of serratus anterior to upper trapezius and serratus anterior to pectoralis major activity are beneficial for selective strengthening programs of the serratus anterior (Ludewig et al, 2004; Piraua et al, 2014). The results of the current study show higher ratio values at $0^{\circ}$ surface tilt than others. For patients with an imbalance of upper trapezius or pectoralis major to serratus anterior activity, exercises that deliver these high ratios would be important components of rehabilitation to enable the selective strengthening of the serratus anterior muscle by mitigating this imbalance (Choi et al, 2017).

The push-up posture utilizes body weight and must be maintained with over-loading when the serratus anterior, as well as other muscles including the upper trapezius and pectoralis major, are activated (Kim and Park, 2018). Imbalanced ratio of serratus anterior and pectoralis major can be disadvantageous for subjects with winged scapula because this lower ratio of serratus anterior and pectoralis major can be associated with shoulder joint pathology (Park et al, 2014). The pectoralis major acts as a synergist muscle, compensating for a weak serratus anterior (Park et al, 2014). In this study, subjects with winged scapula were asked to avoid excessive exercise while participating to familiarized themselves according to surface tilt angles. As a result, although activity of the pectoralis major increased as the surface angle decreased, this was not significant, and since the serratus anterior activity increased substantially at the same time, the ratio of serratus anterior to pec- toralis major was significantly different.

The limitations of this study should be noted. First, only four measuring angles were used and this could be increased for future research. Second, muscles other than the upper trapezius, pectoralis major, and serratus anterior also contribute to the control of the scapula, but they were not considered in this study. Future studies may wish to consider additional muscle groups, larger subject samples with a larger age range, and a broader sample of clinical patients.

\section{Conclusion}

This study found that performing push-up plus exercises on a flat surface with $0^{\circ}$ and $30^{\circ}$ tilt angle achieves high activation of the serratus anterior muscle for selective strengthening. It is therefore recommended that push-up plus exercises on such surfaces are regarded as the best choice for addressing weakness of the serratus anterior muscle and winged scapula. It can also take into account the sequential application, which is first performed at a $30^{\circ}$ and at a $0^{\circ}$ tilt angle for and effective but not $\mathrm{ex}^{-}$ cessive muscle activation.

\section{References}

Choi WJ, Yoon TL, Choi SA, et al. Different weight bearing push-up plus exercises with and without isometric horizontal abduction in subjects with scapular winging: A randomized trial. J BodyW Mov Ther. 2017;21(3):582-588. http://doi.org/10. 1016/j.jbmt.2016.08.018

Cram JR, Kasman GS, Holtz J. Introduction to surface electromyography. NY, USA, Aspen Publishers, 1998.

Decker MJ, Hintermeister RA, Faber KJ, et al Serratus anterior muscle activity during selected rehabilitation exercises. Am J Sports Med. 1999; 
27(6):784-791

Ebaugh DD, McClure PW, Karduna AR. Three-dimensional scapulothoracic motion during active and passive arm elevation. Clin Biomech (Bristol, Avon). 2005;20(7):700-709. http://doi.org/10.1016/ j.clinbiomech.2005.03.008

Kim BG, Gong, WT, Lee SY. The effect of push-up plus exercise with visual biofeedback on the activity of shoulder stabilizer muscles for winged scapula. J Phys Ther Sci. 2010:22(4):355-358. http://doi.org/10.1589/jpts.22.355

Kim TH, Park HK. The comparison for serratus anterior muscle activity during protraction in open chain and closed chain exercises in healthy adults. J KEMA. 2018;2(1):1-5.

Labriola JE, Lee TQ, Debski RE, et al. Stability and instability of the glenohumeral joint: the role of shoulder muscles. J Shoulder Elbow Surg. 2005; 14(1 Suppl S):32S-38S.

Lawrence RL, Braman JP, Laprade RF, et al. Comparison of 3-dimensional shoulder complexkinematics in individuals with and without shoulder pain, part 1: Sternoclavicular, acromioclavicular, and scapulothoracic joint. J Orthop Sports Phys Ther. 2014;44(9):636-645. http://doi.org/10. 2519/jospt.2014.5339

Lear LJ, Gross MT. An electromyographical analysis of the scapular stabilizing synergists during a push-up progression. J Orthop Sports Phys Ther. 1998;28(3):146-157. http://doi.org/10.2519. jospt.1998.28.3.146

Ludewig PM, Hoff MS, Osowski EE, et al. Relative balance of serratus anterior and upper trapezius muscle activity during push-up exercises. Am J Sports Med. 2004:32(2):484-493. http://doi.org/ 10.1177/0363546503258911

Ludewig PM, Phadke V, Braman JP, et al. Motion of the shoulder complex during multiplanar humeral elevation. J Bone Joint Surg Am. 2009;91(2): 378-389. http://doi.org/10.2106/JBJS.G.01483
McClure PW, Michener LA, Sennett BJ, et al. Direct 3-dimensional measurement of scapular kinematics during dynamic movements in vivo. J Shoulder Elbow Surg. 2001;10(3):269-277. http://doi.org/10.1067/mse.2001.112954

Madeleine $\mathrm{P}$, Mathiassen SE, Arendt-Nielsen L. Changes in the degree of motor variability associated with experimental and chronic neck-shoulder pain during a standardised repetitive arm movement. Exp Brain Res. 2008;185(4): 689-698. http://doi.org/10.1007/s00221-007-1199-2

Park KM, Cynn HS, Kwon OY, et al. Comparison of pectoralis major and serratus anterior muscle activities during different push-up plus exercises in subjects with and without scapular winging. J Strength Cond Res. 2014;28(9):2546-2551. http://doi.org/10.1519/JSC.0000000000000443

Park KM, Cynn HS, Yi CH, et al. Effect of isometric horizontal abduction on pectoralis major and serratus anterior EMG activity during three exercises in subjects with scapular winging. J Electromyogr Kinesiol. 2013;23(2):462-468. http://doi.org/10.1016/j.jelekin.2012.11.013

Pirauá AL, Pitangui AC, Silva JP, et al. Electromyographic analysis of the serratus anterior and trapezius muscles during push-ups on stable and unstable bases in subjects with scapular dyskinesis. J Electromyogr Kinesiol. 2014;24(5):675-681. http://doi.org/10.1016/j.jelekin. 2014.05.009

Weon JH, Kwon OY, Cynn HS, et al. Real-time visual feedback can be used to activate scapular upward rotators in people with scapular winging: an experimental study. J Physiother. 2011; 57(2):101-107. http://doi.org/10.1016/S1836-9553 (11) $70020-0$

This article was received September 10, 2019, was reviewed September 10, 2019, and was accepted November 14, 2019. 